\title{
E835 Store Baby Sitting Procedures
}

\section{Control of the RF frequency:}

1. "RF Freq Check" on P85 (E835 Baby Sitter) should be turned OFF.

2. The RF frequency should be adjusted so that it is in the notch of the $4-8 \mathrm{GHz}$ momentum cooling pickup response. The RF frequency device to be controlled depends on which RF system is on. If ARF2 is on, the RF frequency device is A:RLLFS1. If ARF3 is on, the RF frequency device is A:RLLFS0.

IMPORTANT NOTE: A:RLLFS0 and A:RLLFS1 have very different data base scaling (A:RLLFS0 is 4 bytes and A:RLLFS1 is 2 bytes). A:RLLFS0 can be safely knobbed with a mult factor of 1.0 (i.e. no multiplier is required). A:RLLFS1 requires a mult factor of 0.02 or smaller.

The monitoring and adjustment of the RF frequency is accomplished by the following steps:

3. Set up SA1 so that it is connected to CP48-SCH (4-8 GHz momentum cooling pickup). Set the SA center frequency to a harmonic of the RF frequency. This is most easily accomplished by doing one of the following:

- If ARF3 is on, send P41 file 22 to SA1.

- If ARF2 is on, set A:RLLFS0 to the set value of A:RLLFS1 then send P41 file 22 to SA1.

4. SA1 can be viewed on CATV channel pbar 20. If the notch in the momentum cooling pickup response is not at the center frequecy of SA1 adjust the RF. (On the low energy ramp, 1 division on the SA1 display at $5.5 \mathrm{GHz}$ corresponds to $2.3 \mathrm{~Hz}$ in revolution frequency). Once you've made an adjustment to the RF frequency you should reset the SA1 display according to step 3 above.

\section{$E_{c m}$ Tracker Parameters}

The $\mathrm{E}_{\mathrm{cm}}$ tracker of P85 should be turned $\mathrm{ON}$. The current best settings for the $\mathrm{E}_{\mathrm{cm}}$ tracker parameters are the following:

$$
\begin{array}{lll}
\text { Ecm Interval } & =200 \mathrm{sec} \\
\text { Desired Ecm } & =\text { Target } E_{\mathrm{cm}}-\text { the target } \mathrm{E}_{\mathrm{cm}} \text { for the current stack is found at the following URL: } \\
\text { Sigma P Limit } & =2 \mathrm{MeV} \text {. } & 2 \mathrm{Me} \text { :/ cosmo.fnal.gov/commissioning/Present Activities.htm } \\
\text { PU Lower Limit } & =-10 \mathrm{~mm} \\
\text { PU Upper Limit } & =2 \mathrm{~mm} \\
\text { Feedback Gain } & =0.1 \\
\text { Feedback Tc } & =360 \mathrm{sec}
\end{array}
$$

\section{Control of the beam when the E835 target is turned off}

When E835 turns of the gas jet target there are several actions required to prevent a large excursion of the beam energy.

1. Immediately after the Gas Jet is turned off do the following:

- Turn of the momentum cooling (turn off A:CMPS01).

- On P85 (E835 Baby Sitter), turn off the Ecm tracker.

2. If the target will be off for more than $10-15$ min, center the momentum cooling pickups on the beam and turn the momentum cooling on according to the following steps: 
- Set A:RLLFS0 to the current value of A:CENFRQ (if ARF3 is on you should knob it slowly).

- Using P41, send file 22 to SA1.

- Move A:MARAYD so that the notch in the pickup response is at the center frequency of SA1 (1 division is approximately 1200 steps on A:MARAYD). If the notch is left of the center frequency sent negative steps. If the notch is to the right of the center frequency send positive steps. Err on the low frequency side (i.e. leave the notch slightly below the center frequency since the beam will have a tendancy to loose energy over time).

- Raise the setting of A:CMPA01 by $3 \mathrm{~dB}$ and turn A:CMPS01 on.

3. Turn the P85 Ecm tracker back on.

4. Adjust A:CMPA01 to maintain the A:SIGMAP at the desired value.

5. When the target is turned back on:

- Move the momentum cooling pickups so that the notch is one division to the left of the center frequency on SA1 (about +1200 steps on A:MARAYD).

- MAKE SURE YOU TURN THE MOMENTUM COOLING GAIN BACK UP (i.e. restore A:CMPA01 to its original setting). 